\title{
Development of Radiation Hard Particle Detectors Made of Czochralski Grown Silicon
}

\author{
J. HÄRKÖNEN* \\ Helsinki Institute of Physics, Gustaf Hällströmin katu 2, 00014 Helsingin yliopisto, Finland
}

\begin{abstract}
Tracking detectors to be used in the future high-luminosity particle physics experiments have to be simultaneously radiation hard and cost efficient. Magnetic Czochralski silicon wafers can be grown with sufficiently high resistivity (several $\mathrm{k} \Omega \mathrm{cm}$ ) and well-controlled high oxygen concentration. Significant research and development activity aiming to develop particle detectors made of high resistivity magnetic Czochralski silicon has been ongoing during the past decade. Beam test results presented in this paper show that $n$-type magnetic Czochralski silicon strip detectors can be operated with acceptable signal-to-noise ratio at least up to a $1 \mathrm{MeV}$ neutron equivalent $\left(n_{\text {eq }}\right)$ fluence of $1 \times 10^{15} \mathrm{~cm}^{-2}$. The improved radiation hardness, compared to more commonly used float zone silicon, can be explained by the more beneficial electric field distribution inside the detector bulk. We have demonstrated a $\mathrm{S} / \mathrm{N}$ of $>10$ for full-size strip detectors attached to readout electronics at a fluence of $1 \times 10^{15} n_{\text {eq }} / \mathrm{cm}^{2}$. These findings are supporting our transient current technique measurements.
\end{abstract}

DOI: $10.12693 /$ APhysPolA.124.372

PACS: 29.40.Wk, 81.40.Wx

\section{Introduction}

Particle detectors made of silicon are widely used in track finding applications in large high energy physics (HEP) experiments. For instance, a tracker system having a surface of about $220 \mathrm{~m}^{2}$ and 100 million channels has been installed in the compact muon solenoid (CMS) experiment within the CERN Large Hadron Collider (LHC) accelerator [1]. It is obvious that such large-scale scientific instruments must be constructed in a cost-effective and industrial manner. Thus, silicon detectors due to their high precision, radiation hardness and most importantly, their well-established semiconductor manufacturing technology are natural choice for modern HEP instrumentation.

Silicon detectors have traditionally been fabricated on $n$-type high resistivity wafers made by float zone ( $\mathrm{Fz}-\mathrm{Si}$ ) crystal growth technique. Only the $\mathrm{Fz}-\mathrm{Si}$ was previously able to meet the specifications of high resistivity and low impurity level required in radiation detection applications. The high level of purity and consequently low oxygen concentration in Fz-Si, however, is also a drawback since oxygen has experimentally been found to improve the radiation hardness of silicon detectors, as demonstrated by the $R \& D$ work performed by the CERN-RD48 collaboration in 1990's [2]. They developed a concept called DOFZ silicon, which stands for oxygen enrichment by a long lasting oxidation step (e.g. $72 \mathrm{~h}$ at $1150{ }^{\circ} \mathrm{C}$ ) performed before the processing of the detector. It results in an average oxygen concentration in the order of $1 \times 10^{17} \mathrm{~cm}^{-3}$ which is almost one order of magnitude higher than in the standard Fz-Si.

\footnotetext{
*e-mail: jaakko.haerkoenen@cern.ch
}

Alternative way to achieve high oxygen concentration in the detector bulk is simply to use the Czochralski silicon ( $\mathrm{Cz}-\mathrm{Si}$ ) as a substrate material. Nowadays, the Czochralski crystal growth method enables the production of silicon wafers with sufficiently high resistivity and with well-controlled oxygen concentration. Although $\mathrm{Cz}_{\mathrm{Z}-\mathrm{Si}}$ is the basic raw material for microelectronics industry, high resistivity ( $>1 \mathrm{k} \Omega \mathrm{cm}$ ) $\mathrm{Cz}-\mathrm{Si}$ wafers suitable for detector fabrication have become available for HEP community only recently. Application of magnetic field during the crystal growth is a special case of the Czochralski technology. The magnetic field induced the Lorentz force into silicon melt, which reduces the amplitude of the melt fluctuations and thus increases the controllability of the crystal growth process. As a result, erosion of the silica crucible is reduced resulting in less dissolving impurities such as boron or aluminum and consequently, homogeneity of a crystal is improved. This is essential for very large diameter silicon wafers used in CMOS applications. Since particle detectors are large area devices, impurity homogeneity over wafer diameter is also crucial. Thus, the detectors described in this report are made of magnetic Czochralski silicon ( $\mathrm{MCz}-\mathrm{Si}$ ) substrates.

This paper summarizes the R\&D aiming to develop particle detectors made of high-resistivity Czochralski silicon. The motivation behind the developments is to ensure radiation tolerant silicon detectors for the future HEP experiments with very high luminosities [3].

\section{Processing of $\mathrm{Cz}-\mathrm{Si}$ particle detectors}

The high resistivity $\mathrm{Cz}-\mathrm{Si}$ detectors can basically be processed into segmented or pad detectors in the same way as in the case of the traditionally used Fz-Si substrates. The essential difference between $\mathrm{Cz}-\mathrm{Si}$ and $\mathrm{Fz}-\mathrm{Si}$ materials is, however, the oxygen concentration, which requires modified recipes during the back-end process. 
The detectors described in this content were processed at the Microelectronics Center of Helsinki University of Technology starting from 2001. The starting material of the detectors was $4^{\prime \prime}$ single-side-polished $380 \mu \mathrm{m}$ thick $\langle 100\rangle \mathrm{Cz}-\mathrm{Si}$ wafers supplied by Okmetic Oyj. The nominal resistivity of the wafers was $900 \Omega \mathrm{cm}$ and $1.9 \mathrm{k} \Omega \mathrm{cm}$ for $n$ and $p$-type, respectively. Our detector fabrication process contains five mask levels consisting of two thermal oxidations, two ion implantations, and three sputter depositions. A detailed process description as well as device design for large-area $n$-type strip detectors are presented in Refs. [4, 5]. For reference purposes, Fz-Si detectors were processed on Wacker $4^{\prime \prime}$ single-side-polished $300 \mu \mathrm{m}$ thick Fz-Si wafers with resistivity of $1.2-1.3 \mathrm{k} \Omega \mathrm{cm}$.

After the first thermal dry oxidation at $1100^{\circ} \mathrm{C}$ the process wafers were subjected to minority carrier recombination lifetime measurements. The lifetime measurements were done by microwave photoconductivity decay $(\mu \mathrm{PCD})$ method using commercial Semilab WT200 Lifetime scanner [6]. The average and median lifetimes were found to be clearly more than $2000 \mu \mathrm{s}$, which is close to the practical upper limit of the measurement technique, in $\mathrm{MCz}-\mathrm{Si}$ and Fz-Si wafers, both. The lifetime maps and deep level transient spectroscopy spectra, including discussion about crystal growth and process induced contamination sources, are presented in Refs. [7, 8].

After the processing the detector wafers were diced and subjected on capacitance-voltage $(C V)$ and currentvoltage $(I V)$ characterization. The $C V$ measurement reveals the full depletion voltage $\left(V_{\mathrm{fd}}\right)$ as the saturation point of capacitance in voltage axis and the $I V$ measurement provides information about the overall quality of substrates and detector processing. An illustrative example of $C V-I V$ measurements are shown in Fig. 1a and b. The measurements have been done at the room temperature.

As it can be seen in Fig. 1a, the leakage of a $\mathrm{MCz}-\mathrm{Si}$ detector is about $2.5 \mu \mathrm{A}$ at $400 \mathrm{~V}$ reverse bias voltage and about $3 \mu \mathrm{A}$ at $900 \mathrm{~V}$. For a Fz-Si detector, the leakage current is about $0.5 \mu \mathrm{A}$ at $300 \mathrm{~V}$ and about $1 \mu \mathrm{A}$ at $1000 \mathrm{~V}$. The leakage current values are good for both $\mathrm{MCz}-\mathrm{Si}$ and Fz-Si devices. Generally, for HEP applications large area detector is of high quality when the leakage current is less than about $10 \mu \mathrm{A}$ and the detector does not suffer from early current breakdown before or soon after $V_{\mathrm{fd}}$.

\section{Thermal donor formation in $\mathrm{MCz}-\mathrm{Si}$ detector material}

Oxygen thermal donors (TD) are probably one the most studied topics in material physics. It is well known from many studies that the TDs are complexes consisting of four or more oxygen atoms, whose formation takes place during the annealing of silicon wafers at $400-600^{\circ} \mathrm{C}$ $[9,10]$, i.e. at temperatures that are frequently used in the detector processing. Studies based on computational

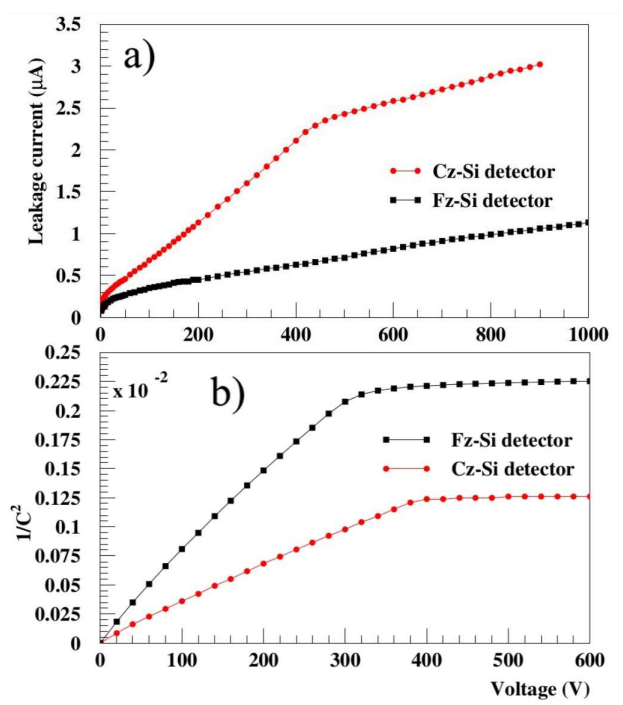

Fig. 1. (a) $I V$ measurements performed on $\mathrm{MCz}-\mathrm{Si}$ (red •) and Fz-Si (black $\square$ ) detectors. The detectors have size $32.5 \mathrm{~cm}^{2}$ and 1024 channels, $6 \mathrm{~cm}$ long strips of $10 \mu \mathrm{m}$ width with $50 \mu \mathrm{m}$ pitch. (b) $C V$ measurements of $\mathrm{MCz}-\mathrm{Si}$ and $\mathrm{Fz}-\mathrm{Si}$ detectors. The capacitance has been plotted in $1 / \mathrm{C}^{2}$ scale in order to visually reveal the $V_{\mathrm{fd}}$, i.e. saturation of capacitance.

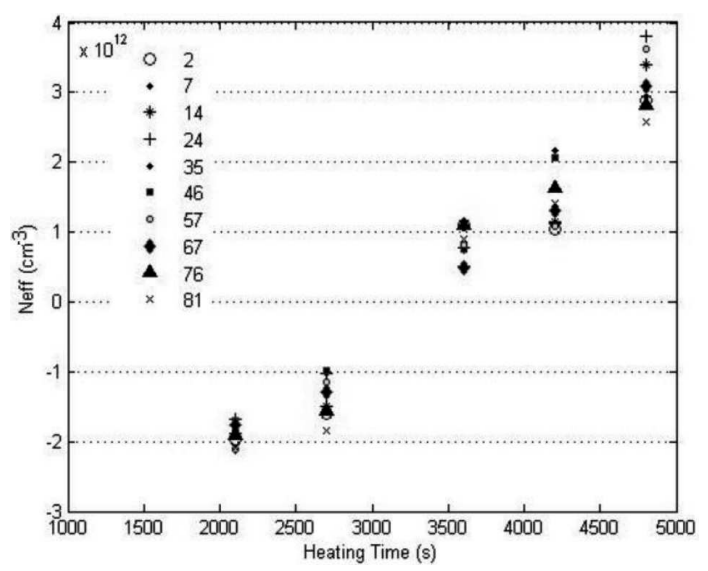

Fig. 2. The effective doping concentration $\left(N_{\text {eff }}\right)$ with respect heating time at $430{ }^{\circ} \mathrm{C}$. Different symbols indicate the detector (size $8 \mathrm{~mm} \times 8 \mathrm{~mm}$ ) location in $100 \mathrm{~mm}$ diameter wafer.

methods suggest that the TD formation and thus shallow donor levels in silicon band gap are due to migration of oxygen complex chains $[11,12]$.

When processing $n$-type $\left(p^{+}\right.$segmentation on phosphorus doped bulk) MCz-Si detectors, the TD formation is generally harmful. TDs result in additional donor doping of the bulk and thus elevated $V_{\mathrm{fd}}$. Thus, temperatures above $400{ }^{\circ} \mathrm{C}$ should be avoided after the last high temperature process step. On the other hand, when processing $p$-type detectors $\left(n^{+}\right.$segmentation on boron doped bulk), it is possible to tailor by acceptor compensation 
the full depletion voltage of the device by deliberate introduction of TDs $[13,14]$. Figure 2 shows the evolution of effective doping concentration $\left(N_{\text {eff }}\right)$ in silicon bulk extracted from detectors by $C V$ method as a function of isothermal annealing time at $430^{\circ} \mathrm{C}$. The material is boron doped, $2 \mathrm{k} \Omega \mathrm{cm} \mathrm{MCz-Si} \mathrm{silicon} \mathrm{[15].}$

It can be seen in Fig. 2 that the $N_{\text {eff }}$ increases almost linearly with respect to heating time at the constant temperature of $430^{\circ} \mathrm{C}$. The $N_{\text {eff }}$ is first negative, i.e. acceptor type of bulk, but inverts to be positive after about 50 55 min annealing indicating that silicon undergoes type inversion from $p$ to $n$.

\section{Radiation hardness of $\mathrm{MCz}-\mathrm{Si}$ detectors}

The radiation hardness of $\mathrm{MCz}-\mathrm{Si}$ detectors has been studied in many irradiation campaigns during the past decade. The irradiations include photons, electrons, neutrons and low and high energy protons from different accelerators [16-19]. The results of these studies can be summarized so that the increase of $V_{\mathrm{fd}}$ in $\mathrm{MCz}-\mathrm{Si}$ material is significantly less than in oxygen-lean Fz-Si detectors when irradiated with charged particles. In case of neutron irradiation, the difference is very small or even non-existent. Since in many HEP applications charged hadrons and neutrons exist simultaneously, there are on-going studies and campaigns of mixed irradiations, but these results are not yet completely understood.

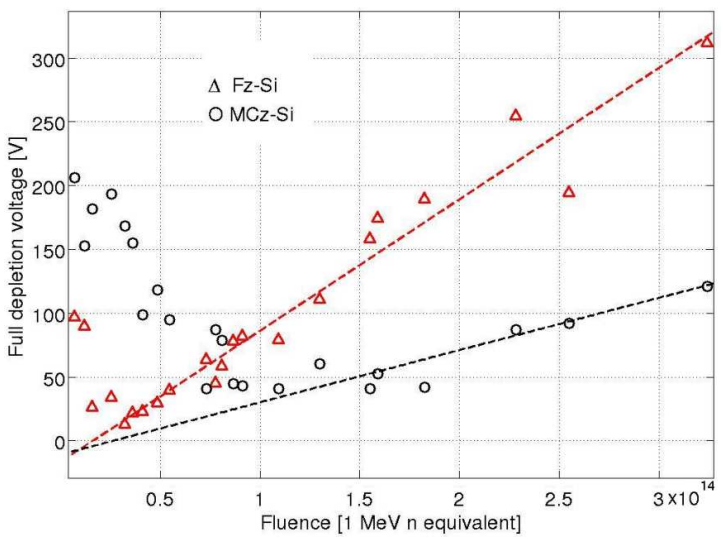

Fig. 3. Evolution of $V_{\mathrm{fd}}$ of $\mathrm{MCz}-\mathrm{Si}(0)$ and $\mathrm{Fz}-\mathrm{Si}(\Delta)$ detectors with respect of irradiation fluence by protons of different energies. The $x$-axis is scaled with appropriate hardness factors into $1 \mathrm{MeV}$ neutron equivalent fluence.

Figure 3 shows evolution of $V_{\mathrm{fd}}$ with respect to proton irradiation fluence. The data points in the graph consist of the measurements made on detectors irradiated with 10, 20, and $50 \mathrm{MeV}$ protons.

As it can be seen in Fig. 3, the increase of $V_{\mathrm{fd}}$ with respect to proton irradiation fluence is much less for $\mathrm{MCz}-\mathrm{Si}$ detectors than of $\mathrm{Fz}-\mathrm{Si}$ detectors produced with the same design and processing, and irradiated under the same conditions.

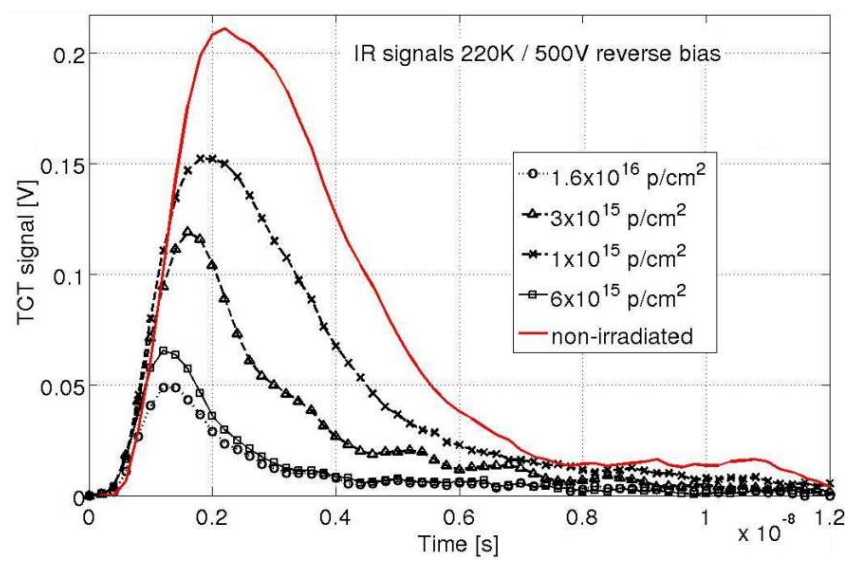

Fig. 4. IR signals of $24 \mathrm{GeV} / \mathrm{c}$ irradiated $\mathrm{MCz}-\mathrm{Si}$ detectors with respect of non-irradiated reference detector. The reverse bias during the measurement was $500 \mathrm{~V}$ and the temperature $220 \mathrm{~K}$.

The current transient response of $24 \mathrm{GeV} / \mathrm{c}$ proton irradiated $\mathrm{MCz}-\mathrm{Si}$ detectors is shown in Fig. 4. The transients are recorded by using a transient current technique (TCT) setup and by illuminating the detectors by short infrared (IR) laser pulses [20-22]. The pulse length of IR excitation is about $30-50 \mathrm{ps}$ and the wavelength is $1060 \mathrm{~nm}$. Under these conditions, the light absorption in silicon is almost homogeneous through the entire $300 \mu \mathrm{m}$ thickness of a detector. Thus, IR TCT measurement is an effective way to simulate the effects of incoming minimum ionizing particles (MIPs).

When assuming $100 \%$ charge collection efficiency (CCE) for a non-irradiated detector, one can see that the sample irradiated with $1 \times 10^{15}$ protons $/ \mathrm{cm}^{2}$ still operates with CCE exceeding 50\%. After more irradiation, the collected signal (i.e. integral of current over time) decreases rapidly. The cause for this is that the $300 \mu \mathrm{m}$ thick detector cannot be anymore maintained in full depletion, as indicated in Fig. 3, and due to excessive trapping by radiation induced defects. This is clearly visible in Fig. 5, which shows collected charge with respect to irradiation fluence.

The data presented in Fig. 5 is obtained by IR-TCT method, whose principle is illustrated in Fig. 4. The detectors have been irradiated with protons of energies $9 \mathrm{MeV}$ and $24 \mathrm{GeV}$. It can be seen in Fig. 5 that the collected charge drops below 5000 electrons soon after $2.5 \times 10^{15} n_{\mathrm{eq}} / \mathrm{cm}^{2}$ irradiation fluence. About 5000 electrons signal is a practical minimum threshold for detectors attached to readout electronics in the HEP experiments.

\section{Operation in high-intensity particle beam}

In order to prove the concept, it is important to verify the functionality of novel detectors under realistic operating conditions. During the past decade, many test 


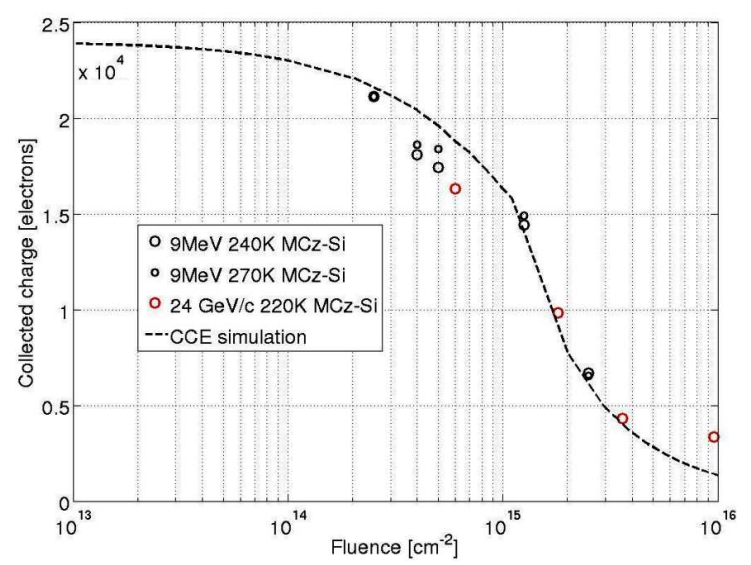

Fig. 5. Experimental data points and simulation of MCz-Si detectors. The simulation assumes $220 \mathrm{~K}$ temperature and $500 \mathrm{~V}$ operating voltage. The experimental data points are based on IR-TCT measurements.

beam experiments were carried out for $\mathrm{MCz}-\mathrm{Si}$ detectors at CERN H2 beam area utilizing $220 \mathrm{GeV} / \mathrm{c}$ muon beam from Super Proton Synchrotron (SPS) accelerator [23-25]. The characterization setup utilized in these test is Silicon Beam Telescope (SiBT) [26]. The SiBT is an essential test and measurement platform to characterize full size detector systems in real beam-like conditions with appropriate readout electronics and data acquisition (DAQ) system. The beam telescope consists of eight reference planes placed in a Peltier element cooled cold box. The reference detectors, providing track information of incident particle beam, are commercially available and made of Fz-Si sensors produced by Hamamatsu Photonics K.K. (HPK).

The DAQ and off-line analysis of the telescope are based on CMS software and analysis framework and are described in detail in Refs. [26, 27]. The output of the DAQ is expressed in analog-digital-counts (ADC), i.e. arbitrary units. For the conversion into absolute charge units we assume that the non-irradiated reference planes operate with $100 \%$ relative CCE and their maximum collected charge is in average $40 \mathrm{ADC} \pm 2 \%$. Thus, taking into account minimum ionizing particle (MIP) ionization in silicon, 80 electron equivalent charge $\left(e^{-}\right)$per $\mu \mathrm{m}$, then one ADC would correspond equivalent of about

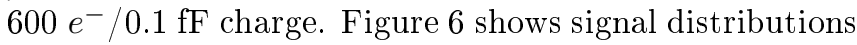
of MCz-Si (DUT) and one reference plane, showing highest signal among the eight planes. The data has been recorded during the same data taking session and both sensors were biased with $150 \mathrm{~V}$. The temperature of the cold box during the data taking was set to $-20^{\circ} \mathrm{C}$.

It can be seen that the most probable value (MPV) of the signal from non-irradiated $\mathrm{MCz}-\mathrm{Si}$ detector is slightly above $40 \mathrm{ADC}$, i.e. the same value, which is seen from eight reference planes. The second signal distribution, corresponding to irradiation fluence $6 \times 10^{14} n_{\mathrm{eq}} / \mathrm{cm}^{2}$, the MPV is about $30 \mathrm{ADC}$, i.e. $75 \%$ relative $\mathrm{CCE}$ corre-

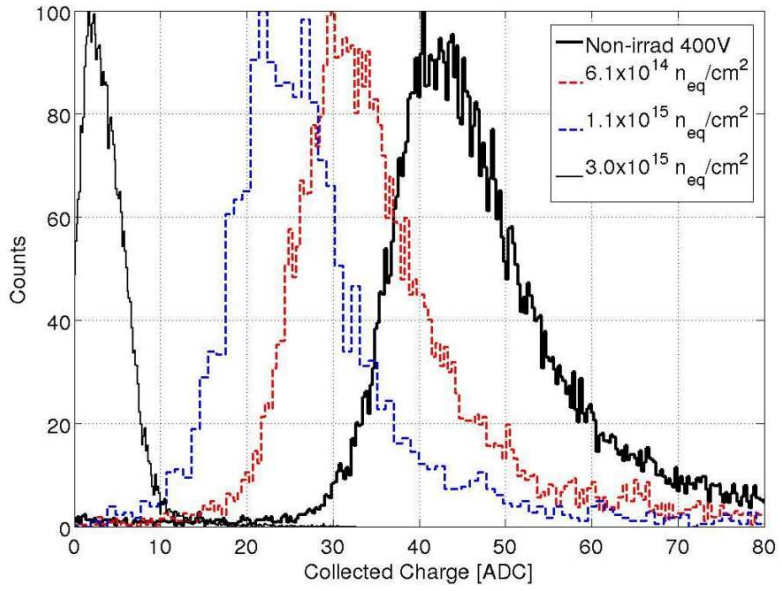

Fig. 6. Superimposed cluster charge distributions for four of the $n$-type $\mathrm{MCz}-\mathrm{Si}$ detectors. The distributions have been scaled so that there are 100 counts in the highest bin.

sponding to about 18000 electron equivalent charge. It is notable that it is in good agreement with IR-TCT data points shown in Fig. 5. The third distribution corresponds to $1.1 \times 10^{15} n_{\mathrm{eq}} / \mathrm{cm}^{2}$ fluence and MPV is clearly more than 20 ADC, i.e. more than 50\% CCE [28]. The fourth distribution correlates with three times higher fluence and the collected charge is only about $7 \mathrm{ADC}$, i.e. about $4000 e^{-}$, which is not anymore sufficient for appropriate signal-to-noise ratio (SNR).

\section{Summary}

The $\mathrm{MCz}-\mathrm{Si}$ material has been studied extensively in the RD50 and RD39 collaborations and it has been found to be more radiation hard against protons than traditional Fz-Si or diffusion oxygenated silicon (DOFz-Si). Furthermore, since $\mathrm{Cz}-\mathrm{Si}$ is commonly used semiconductor material in the microelectronics industry, it is available in large quantities and many commercial foundries are familiar with its processing. These aspects are important, when constructing large tracking systems that can consist of hundreds of square meters of silicon sensors. During the processing, however, one has to put special emphasize on the formation of oxygen donors and the temperature range of TD formation should be avoided.

The beam test results show that the produced $\mathrm{MCz}-\mathrm{Si}$ sensor have performance comparable to commercially mass produced strip sensors delivered earlier for a large-scale particle physics experiment. The signal-to-noise ratio of a non-irradiated $\mathrm{MCz}-\mathrm{Si}$ sensor exceeds 40 when biased with appropriate operating voltage. $1 \times 10^{15}$ is the radiation hardness design target of strip detectors to be used in the future in the upgraded LHC experiments. The beam test results show that the MCz-Si detectors operate with clearly more than 50\% CCE and 10 SNR after this fluence. 
$\mathrm{CCE}$ is a complex product of the trapping and electric field distribution $E(x)$ in heavily irradiated silicon. According to the existing knowledge, the trapping cannot be influenced by engineering of silicon material properties. Thus, the only way to improve the radiation-hardness is to optimize $E(x)$. In $\mathrm{MCz}-\mathrm{Si}$ detectors part of the electric field remains on the segmented front side of the detector, while in Fz-Si detectors electric field maximum shifts completely on the rear side. Having a strong or at least moderate electric field near collecting junction is beneficial for the detector charge collection efficiency [24, 29].

By the date, elevated oxygen concentration is the only successfully demonstrated method. The essential difference between the $\mathrm{MCz}-\mathrm{Si}$ and $\mathrm{Fz}-\mathrm{Si}$ is the oxygen concentration. It is possible to enhance the oxygen concentration in Fz-Si by prolonged high temperature oxygen diffusion, which might last up to hundreds of hours. The compromise is, however, increased processing costs, limited number of qualified processing vendors, as well as risk of induced contamination. In closing, strip detectors made of six inch diameter high-resistivity $\mathrm{MCz}-\mathrm{Si}$ wafers stand as mass production feasible and cost-effective candidate for future HEP experiments requiring ultimate radiation hardness.

\section{References}

[1] The C.M.S. Collaboration, J. Instrum. 3, S08004 (2008).

[2] G. Lindström, et al., Nucl. Instrum. Meth. Phys. Res. A 465, 60 (2001).

[3] R\&D Proposal - Development of radiation hard semiconductor devices for very high luminosity colliders, LHCC 2002-003/P6, 15.2.2002.

[4] J. Härkönen, et al., Nucl. Instrum. Meth. Phys. Res. A 514, 173 (2003).

[5] E. Tuovinen, Ph.D. Thesis, Helsinki University of Technology, 2008.

[6] Recombination Lifetimes in Silicon, Recombination Lifetime Measurements in Silicon, Eds. D.K. Schroder, D.C. Gupta, F.R. Bacher, W.M. Hughes, American Society for Testing and Materials, ASTM STP, Philadelphia 1998, p. 1340.

[7] J. Härkönen, E. Tuovinen, P. Luukka, E. Tuominen, K. Lassila-Perini, J. Nysten, Phys. Scr. T 114, 88 (2004).

[8] J. Härkönen, E. Tuovinen, Z. Li, P. Luukka, E. Verbitskaya, V. Eremin, Mater. Sci. Semicond. Proc. 9, 261 (2006).
[9] G.S. Oehrlein, J. Appl. Phys. 54, 5453 (1983).

[10] K. Wada, Phys. Rev. B 10, 5884 (1984).

[11] Y.J. Lee, J. von Boehm, M. Pesola, R.M. Nieminen, Phys. Rev. B 65, 085205 (2002).

[12] Y.J. Lee, J. von Boehm, M. Pesola, R.M. Nieminen, Appl. Phys. Lett. 82, 2094 (2003).

[13] J. Härkönen, E. Tuovinen, P. Luukka, L. Kauppinen, Z. Li, M. Moll, A. Bates, K. Kaska, Nucl. Instrum. Meth. Phys. Res. A 552, 43 (2005).

[14] M. Bruzzi, D. Menichelli, M. Scaringella, J. Härkönen, E. Tuovinen, Z. Li, J. Appl. Phys. 99, 093706 (2006).

[15] E. Tuovinen, J. Härkönen, P. Luukka, E. Tuominen, Mater. Sci. Semicond. Proc. 10, 179 (2007).

[16] E. Tuominen, J. Härkönen, P. Luukka, E. Tuominen, E. Tuovinen, I. Riihimäki, A. Virtanen, IEEE Trans. Nucl. Sci. 50, 1942 (2003).

[17] Z. Li, J. Härkönen, W. Chen, J. Kierstead, P. Luukka, E. Tuominen, E. Tuovinen, E. Verbitskaya, V. Eremin, IEEE Trans. Nucl. Sci. 51, 1901 (2004).

[18] S. Dittongo, L. Bosisio, D. Contarato, G. D'Auria, E. Fretwurst, J. Härkönen, G. Lindström, E. Tuovinen, Nucl. Instrum. Meth. Phys. Res. A 546, 300 (2005).

[19] E. Tuovinen, et al., Nucl. Instrum. Meth. Phys. Res. A 568, 83 (2006).

[20] V. Eremin, N. Strokan, E. Verbitskaya, Z. Li, Nucl. Instrum. Meth. Phys. Res. A 372, 388 (1996).

[21] B. Dezillie, V. Eremin, E. Verbitskaya, Z. Li, Nucl. Instrum. Meth. Phys. Res. A 452, 440 (2000).

[22] J. Härkönen, et al., Nucl. Instrum. Meth. Phys. Res. A 581, 347 (2007).

[23] P. Luukka et al., Nucl. Instrum. Meth. Phys. Res. A 568, 72 (2006).

[24] P. Luukka, et al., Nucl. Instrum. Meth. Phys. Res. A 604, 254 (2009).

[25] L. Spiegel, et al., Nucl. Instrum. Meth. Phys. Res. A 628, 242 (2011).

[26] T. Mäenpää, et al., Nucl. Instrum. Meth. Phys. Res. A 593, 523 (2008).

[27] T. Mäenpää, M.J. Kortelainen, T. Lampén, IEEE Trans. Nucl. Sci. 57, 2196 (2010).

[28] P. Luukka, et al., Nucl. Instrum. Meth. Phys. Res. A 612, 497 (2010).

[29] E. Tuovinen, J. Härkönen, P. Luukka, T. Mäenpää, H. Moilanen, I. Kassamakov, E. Tuominen, Nucl. Instrum. Meth. Phys. Res. A 636, 79 (2011). 